Document downloaded from:

http://hdl.handle.net/10251/50445

This paper must be cited as:

Catalá Bolós, A.; Jaén Martínez, FJ.; Martínez-Villaronga, A.; Mocholi Agües, JA. (2011). AGORAS: Exploring Creative Learning on Tangible User Interfaces. En Computer Software and Applications Conference (COMPSAC), 2011 IEEE 35th Annual. Institute of Electrical and Electronics Engineers (IEEE). 326-335. doi:10.1109/COMPSAC.2011.50.

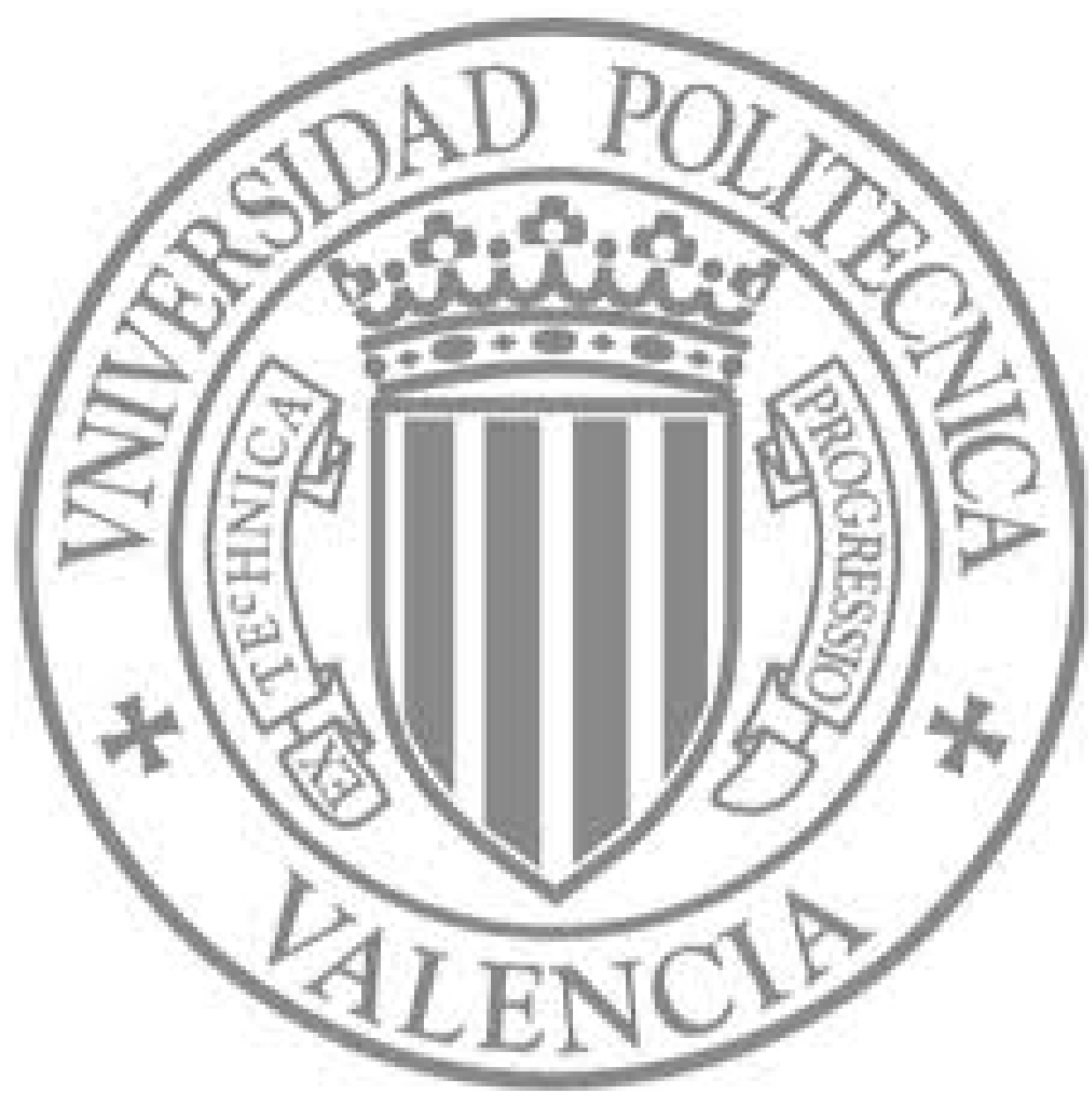

The final publication is available at

http://dx.doi.org/10.1109/COMPSAC.2011.50

Copyright Institute of Electrical and Electronics Engineers (IEEE) 


\title{
AGORAS: Exploring Creative Learning on Tangible User Interfaces
}

\author{
Alejandro Catala, Javier Jaen, Adria A. Martinez-Villaronga, Jose A. Mocholi \\ ISSI Group, Departamento de Sistemas Informáticos y Computación (DSIC) \\ Universitat Politècnica de València \\ Camino de Vera s/n, 46022 Valencia, Spain \\ \{acatala, fjaen, admartinez, jmocholi\}@dsic.upv.es
}

\begin{abstract}
Departing from creative learning foundations, this paper discusses on the suitability of interactive tables as a grounding technology to support creative learning for several reasons: support for social learning, because the subjects share a physical space as in traditional non-digital technologies; communication during the creative, experimental and reflexive process is direct and not computer-mediated; and subjects can carry out the task in parallel on the same surface. Considering reflection, discussion and creation processes in a loop, an experiment with teenagers has been conducted comparing a digital-based against a pure tangible tabletop in a task of creating entities consisting of blocks and joint elements. This preliminary study, designed to obtain initial insights about whether the grounding technology may become a promising tool to support creative learning, explores some aspects such as productivity, complexity of designs and concurrent comanipulation. The results showed that subjects were more productive in terms of the number of solutions obtained using the non computer-mediated approach. However using the digital tabletop approach subjects design, on average, more complex or elaborate solutions in terms of the number of involved bodies and joints. Finally, an important finding was that teams established more frequently concurrent cooperation schemes in the digital tabletop condition by sharing more effectively the creation space.
\end{abstract}

Keywords-Interactive Surface; Tabletop Displays, Tangible User Interface (TUI); Creativity

\section{INTRODUCTION}

Recent studies have shown that nearly $30 \%$ of Spanish secondary school students do not manage to terminate this stage of their education successfully [1]. The situation in most EU countries, however, is not optimal $14.4 \%$ average drop-out rate (over 6 million youngsters). As it is discussed in [2], empirical evidence is not conclusive about the effectiveness of educational policies on student performance but, as Cropley points out in [3], traditional educational systems, which too often assume the existence of only one correct or at least one preferred answer to any problem, strongly inhibit innovation processes and the generation of new ideas. This means that a creative pupil, or one with a different cultural background, is often considered as a source of distortion and distraction in the process of acquiring the knowledge that the teacher desires to impart.

In spite of this classical approach towards education, we are convinced that the combination of interactive technologies, constructivist methods and appropriate interfaces, have a great potential for improving creative education by developing the three factors regarded by Amabile [4] as necessary for creative learning (knowledge, creative thinking and motivation). Although several technological approaches have been used to support to a greater or lesser degree the pedagogical factors that promote creativity, none of the existing computer-based learning approaches, as it will be discussed later; have succeeded in supporting effectively creative learning scenarios.

However, recent advances in the HCI research field have resulted in new forms of interaction known as Tangible User Interfaces (TUIs). In the case of TUIs such as interactive tables, users interact with the system with their hands and their fingers and also by manipulating specially configured physical objects. The concept goes thus far beyond the simple idea of multi-touch. This type of tables means much more than flat screens that can detect many fingers simultaneously. Interacting with the fingers still belongs to the idea of pointing devices, while interacting with physical objects can take us much farther. Such objects can represent abstract concepts or real entities; they can relate to other objects on the surface; they can be moved and turned around on the table surface and all these spatial changes can affect their internal properties and relationships with neighboring objects. As a result, these interfaces strengthen concepts such as "social interaction" and "collaboration" [5][6][7] or "game interaction".

In this paper a part of AGORAS is presented. It is a new learning environment based on tabletops to support creative learning and one of its components that enables the creation of embodied entities for reactive $2 \mathrm{D}$ ecosystems is analyzed. This preliminary study compares the performance in terms of creativity factors of our interactive tabletop approach with respect to an equivalent traditional learning environment based on purely physical objects. The goal is to obtain initial insights about whether this technology is a promising tool in the field of creative learning that needs further research and development.

The paper is organized as follows, Section 2 defines the concept of creativity, the different existing technological 
approaches that have been used to support learning and the reasons why we believe TUIs are an ideal candidate for further research. Section 3, describes the experimental conditions under which present research was performed and the results obtained and, finally, Section 4 presents the main conclusions and future work.

\section{CREATIVE LEARNING AND INTERACTIVE TABLETOPS}

In spite of the wide variety of opinions about how creativity should be defined and the fact that the study of theoretical frameworks for defining creativity is a line of research that is still in its early development, the report "Assessing Creativity: A Guide for Educators" from the National Research Center on the Gifted and Talented provides detailed categories of the different approaches [8]. Among these, the proposal by Teresa Amabile stands out for its simplicity and capacity to join together aspects that had been suggested separately in other approaches [4]. According to Amabile, creativity arises as a result of the combination of three factors: knowledge, creative thought and motivation.

Knowledge consists of all the information possessed by individuals to solve a problem. Howard Gardner identifies two types: knowledge relating to a profound understanding of a certain domain (in-depth understanding) and a more superficial understanding of multiple areas (in-breadth understanding) [9].

Creative thinking can be summed up according to the model proposed by Amabile as the presence of the following individual abilities to a greater or lesser degree: the ability to disagree with others and experiment with different solutions to proposals chosen by the majority; the ability to persevere in difficult or problematic situations, and, finally, the ability to gestate ideas during periods in which one alternately forgets the problem and returns to it with a new perspective.

Finally, a great number of researchers consider motivation as the most important factor in the development of creativity. Among others, Amabile, Gardner and Sternberg stress the prevalence of intrinsic motivation, which is directed by interest, satisfaction and the challenges presented in order to solve a problem, over "external pressures" or extrinsic motivation. As a result and as pointed in [10], in order to give way to the so-called creative society, some substantial advances in the role technology is playing in education are needed.

If the different technological supports currently used in both traditional and modern learning applications are reviewed, and how they support to a greater or lesser degree are analyzed, the pedagogical proposals for promoting creativity show a great diversity of approaches. Some of them are reviewed next.

Offline multimedia educational environments, typically in the form of CDs or DVDs, are interactive and allow learning methods to be defined which the user must comply with. Although these systems focus on learning through creating artifacts, which is an important element in creativity, interaction is limited to one user, or perhaps two sharing the same application. This limits the divergent and convergent thought processes, which Scott, Leritz and Mumford [11] considered important.

Web-based educational environments, on their side, tend to bring learning management systems a little further and may allow tutors to follow students' progress. Pupils can share impressions and opinions with classmates and discuss the activities, thus encouraging critical and divergent thinking. However, activities are in general based on classical instruction and when oriented towards creativity they are usually in the form of individual activities.

Mobile device based educational environments involve much more than simply adapting resources and applications to their small-scale interface, and depend also on the connection and the area of use. Activity design can incorporate learning based on locating the available contextual information. Social networks can be used in search of cooperation, using mobile attributes in a literal sense, and they can be provided with "just in time" information systems. The limitations of this type of environment are those imposed by the interaction limitations of the devices themselves and by the fact that virtual information cannot be easily handled collectively, since it is presented in the individual phones of each member. This greatly limits the type of creative action that can be performed with this technology.

Educational environments based on robots and mechanical elements, are programmable electronic devices with sensors and actuators. The concept of programmable blocks in combination with the Logo language and the Lego construction kit are especially important [12][13]. These systems inspired the construction kits PicoCricket [14], which permits artistic creations with lights, sound, music and movements, and Lego MindStorms NXT [15] designed for the construction of robots. RoboCup [16] is another educational initiative in the use of robots, involving diverse competitions in various categories. Here again, creative learning is fostered by the construction of physical objects. Currently, the main disadvantages of these systems are their high cost, low degree of interaction (the robots have a limited number of pre-defined movements), and for inexperienced users they are still difficult to program.

After an overview of how information technologies are currently applied in the context of education, it can be affirmed that although they all bring some elements that can boost at least several of the three pillars on which creative learning is built (knowledge, creative thought and motivation), these systems do hardly achieve the cooperation and group participation that is so often present in many non technological activities, such as gaming, which, since infancy, constitute an essential part of our initial learning stage. Moreover all these instructional system are usually developed in an ad hoc way with a specific learning domain in mind. What we thus propose is the creation of a platform that will profit as much as possible from traditional nontechnological gaming activities, allowing pupils to create their own games according to the rules they themselves will lay down, and which, by means of digital technologies, will provide stimulating environments in which pupils will be 
able to experience interactively the results of their design decisions.

In this sense it is believed that Tangible User Interfaces may play an important role in the development of such type of environments. TUIs combine control and representation in a single physical device [17]. These interfaces strengthen concepts such as "social interaction" and "collaboration" [5][7] or "game interaction" [18] and it has also been shown that this type of infrastructure is ideal for exploratory and creative activities and allows users to create constructions that would be impossible by other means [6]. They encourage intrinsic motivation and provide optimal learning experiences in which users are motivated to learn the effects of an action on the behavior of the interactive world by manipulating tangible elements. This has been recently showed in different areas such as music creation and performance [19][20] or Logo programming [21]. We are convinced that this type of exploratory interfaces will constitute the ideal platform for the non-trivial tasks involved in game definition and programing, such as the definition of the reactive behaviour, workflows and choreographies of the game involved entities, and the relations and interactions between them.

In this line of research a new framework known as AGORAS (Augmented GeneratiOn of Reactive Ambients on Surfaces) is proposed to support the creation and simulation of reactive gaming scenarios on $2 \mathrm{D}$ interactive surfaces. AGORAS is a model-driven environment [22] in which users may define their own types of reactive entities, their properties, behaviors, the number of instances of each type and the reactive rules that govern the ecosystem. In this way, students can create different solutions to proposed problems and evaluate the adequacy of their creations. To support these activities several editing tools have been created. Among them, in this paper special attention is paid to a tabletop-based editor of the physical properties of entities. In AGORAS, the embodiment of entities is defined by using basic shapes known as bodies that are joined together by means of different types of joints. In this way, users may define any type of articulated entity that will exhibit natural physical behaviors such as motion, acceleration and

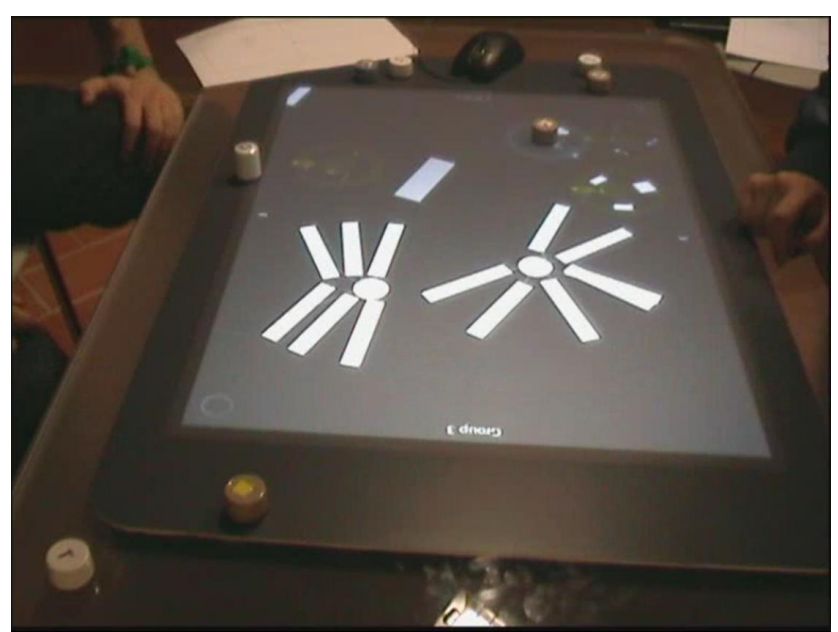

Figure 1. Example of edition process of an articulated entity. collisions when simulated with our physics engine (see Fig. 1). The embodiment and physical properties of entities are created by tangible means using knobs and multi-touch gestures in a shared space in which several users may collaborate.

Because this constructive process is a creative task that users have to perform, it is a valuable scenario to obtain initial insights about the adequacy of interactive tabletops to support creativity.

\section{EXPERIMENT ON CREATING STRUCTURES}

A preliminary evaluation of the AGORAS editor of structures for entities has been designed to know how it performs when compared with another classical learning approach based on using purely tangible elements with no computer-based support. The goal is to know how fundamental creativity attributes such as collaboration, communication and the complexity of the obtained solutions are affected when using an interactive tabletop approach. The choice of a pure tangible approach and not one based on desktop computers as an element for comparison is to have two interaction systems that are as similar as possible in terms of user involvement and participation. If a WIMP (Window-Icon-Menu-Pointers) application running on a desktop had been used, users would have had a serious disadvantage over those using an interactive table because the interaction in the first case is mediated by a single mouse which is a centralized and exclusive device. Therefore it would not have obtained comparable interaction situations in which both members of the team have the same chances of interacting with the system. As a result comparisons about the degree of collaboration, participation, and communication patterns would have not been valid.

\section{A. Participants}

Fourteen teenagers participated in the study, 8 male and 6 female, all students from several secondary local schools. Two participants were left-handed and one was ambidextrous. Ages ranged from 15 to 18 ( $\mathrm{M}=16.6$, $\mathrm{SD}=0.9$ ). Eight participants reported using a personal computer every day, three almost every day, and three

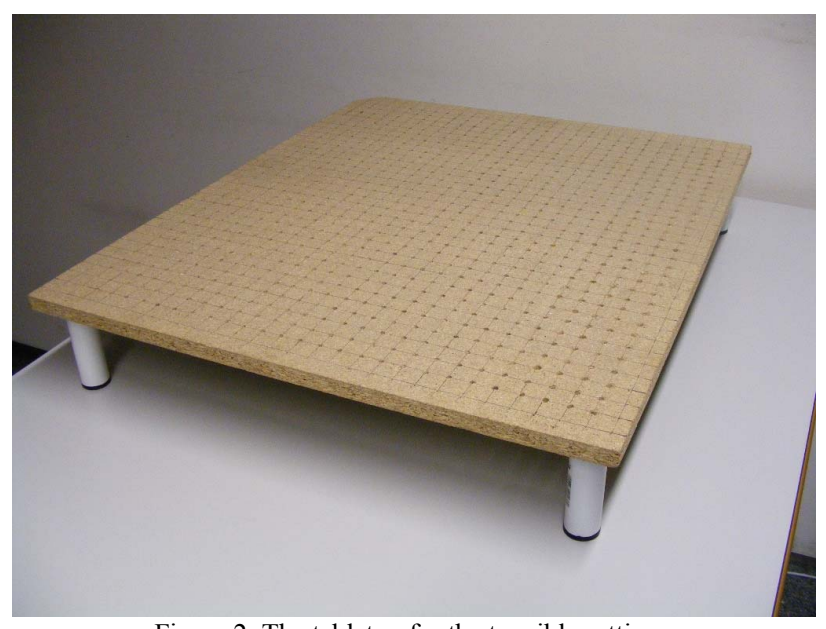

Figure 2. The tabletop for the tangible setting. 
reported using one once or twice per week. Eleven participants reported themselves to be regular users of touchenabled devices, whereas three had seldom or never used one. No one had any previous experience of surface computers.

Participants took part of an extramural short course about new and emerging technologies. The goal of the course was to motivate teenagers to study core subjects in the curriculum such as physics and computing. The course was organized by a clubhouse dependent on the Education \& Culture section of a local city council, which regularly offers courses targeted to teenagers. Since the enrollment was completely free and voluntary, and it is a clubhouse, not a school, there was not any kind of participant pre-selection according to school performance profiles.

\section{B. Equipment and Instrumentation}

Two different platforms were designed and implemented to support the experiment. As described in more detail below, one was a fully tangible approach based on building blocks, and the other was a digital version based on surface computing. The digital version is actually a reduced modification of the entity editor of AGORAS requiring some changes to implement the experiments, basically facilitating the management of the experimentation as switching between participants' workspaces.

Solution forms printed in paper sheets were used by participants to report and explain every solution by means of a sketch and annotations before its implementation in the experimentation platforms. Two video cameras were used to record the sessions to support video analysis later. Colored cards and pucks were used to identify participant groups and switch between their workspaces. Also colored strips were used around wrists in order to identify participants' hands in the video.

\section{1) Tangible Tabletop Platform}

A tangible platform has been constructed from typical hardware pieces. This is made of a conglomerate tabletop with dimensions $590 \times 700 \mathrm{~mm}$. with a regular grid of $28 \times 32$ holes on it with separation of $2 \mathrm{~cm}$. between them (see Fig.

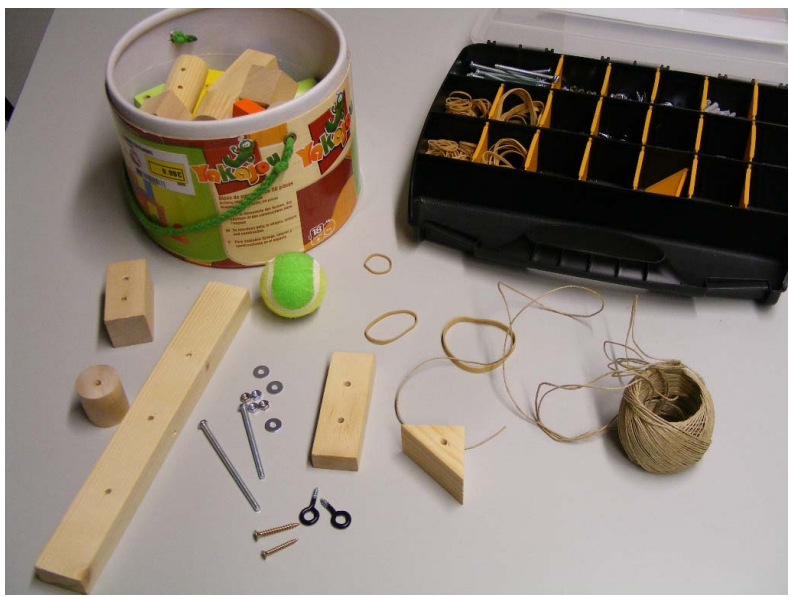

Figure 3. Wooden building blocks and joint elements for the tangible setting.
2). These are for fixing building blocks to the surface if needed as explained below. The tabletop has four legs to keep it in horizontal position, but if needed a stand is also used to configure it as a slanting plane.

Several types of building blocks are available. They are basically wooden building blocks from construction play sets for children which have been drilled. They consist of cylinders, boxes, cubes and triangular prisms, which seen from a perpendicular point of view to their faces would describe circled, rectangular, squared, and triangular shapes (see Fig. 3).

The joint elements used to keep blocks fixed or to create movable constructions are basically based on short strings, elastic bands, screws, hooks, nuts and bolts (see Fig. 3). By combining several blocks and joint elements more complex joints and other functional components can be assembled. For instance an elbow joint, a revolute joint or even a catapult could be assembled as shown in Fig. 4.

This tangible platform allows the construction of any kind of fixed or articulated components based on basic rigid bodies and joints as described above. As this platform is completely tangible and physical, it is subject to actual physics, and the constructions are arranged in the space (i.e. 3D). Users have a high number of pieces of each type at hand in a bucket and they only have to grasp them as needed.

\section{2) Digital Tabletop Platform}

The digital platform is based on an interactive surface, in particular a Microsoft Surface unit. Several tangible tagged pucks (see Fig. 5) are used as input tools and controllers that expand interaction with fingers.

The software application is developed on top of the Microsoft XNA framework and the Farseer physics engine [23], using the Microsoft Surface SDK v1.0. This application supports the creation of $2 \mathrm{D}$ worlds that allows recreating what the tangible tabletop approach does. In this sense, since the digital approach is confined to the plane of the surface, the building blocks counterparts are actually $2 \mathrm{D}$ shapes (i.e. circles, rectangles, squares and triangles) as illustrated in Fig. 6 , although conserving the actual dimensions.

There are also counterparts for the joints in the digital

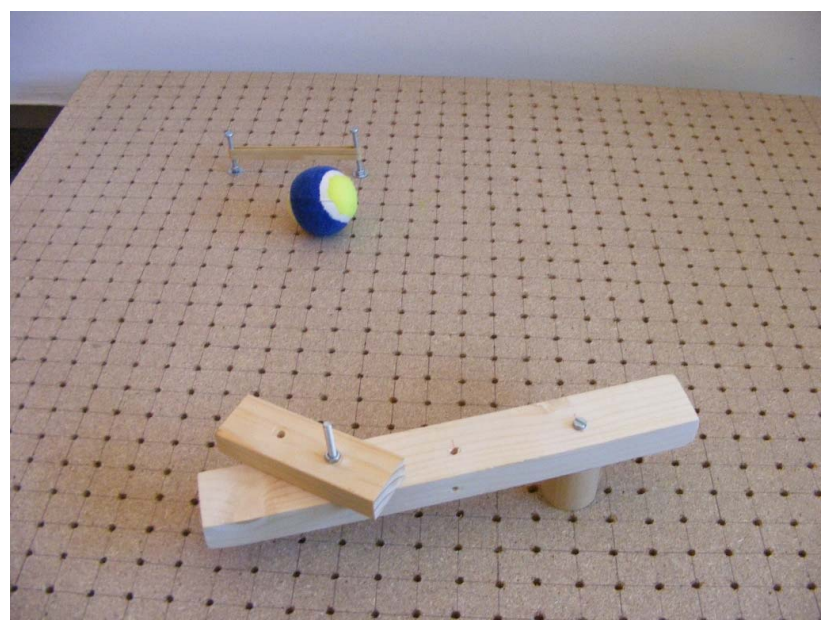

Figure 4. Example of joints and block assemblies. 


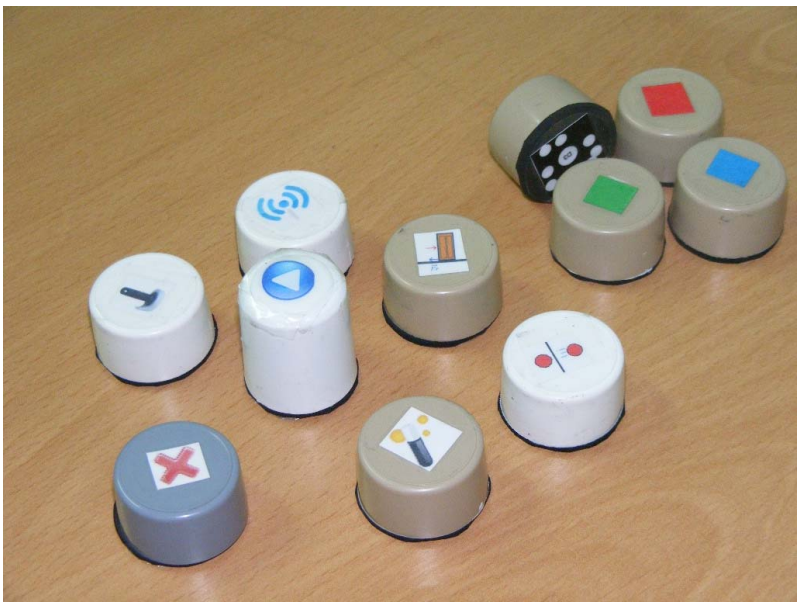

Figure 5. Tagged pucks used as input tools and controller in the digital setting.

version, although the correspondence is not necessarily oneto-one because joints can be simplified in the virtual world. For example, if one needs to join a block to a point distant from its position in the plane, there are two basic cases in the tangible approach: in the case of requiring the block being fixed to the plane, the screw crossing the block used to fix it to the plane could be used to tie a string from it to the desired distant point where there is a bolt; if requiring the block to be freely movable, a hook should be driven into the block, and get the string tied to it and the bold in the distant point. However, in the digital version just a pin joint would allow to reach both cases but some other mechanism, either a tool or a joint should be used in addition to fix the block to the surface. Thus, several digital joints are available (see Fig. 6): a fixed pin joint allows joining a block to a specific location in the surface by means of a string; a regular pin joint allows joining two blocks by means of a string; an elastic joint behaves as a pin joint but using an elastic band instead of a string.

When editing a world, the action of grasping a block from the bucket or a joint element from the toolbox is performed by using the "creation" puck. This is a tagged tangible tool with a "magic wand" as icon (see Fig. 5). It

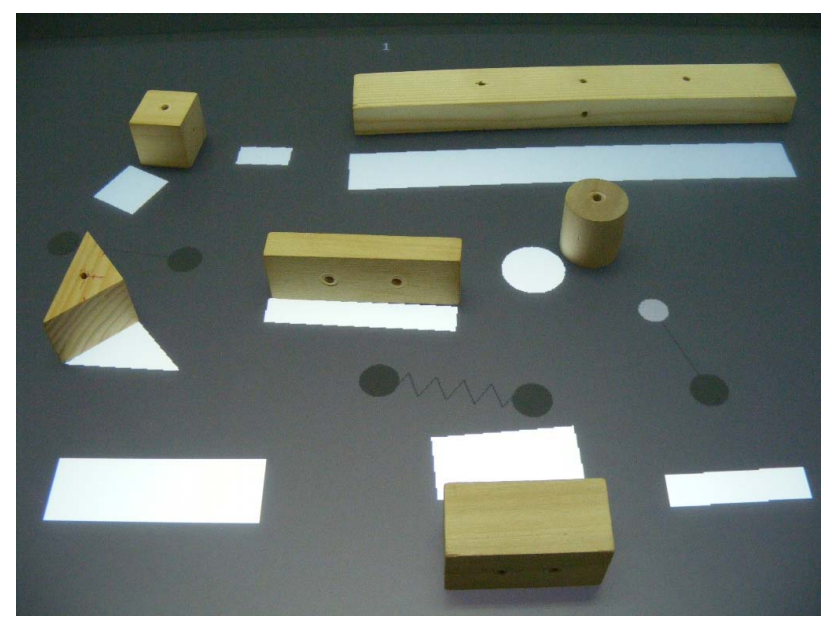

Figure 6. Building blocks and joint elements for the digital setting. gives access to the menus of block and joint types. The selection of a specific element adds and leaves an instance in the surface. Users can then manipulate each block or joint as required by means of finger-based interaction. If an element is to be removed, an "eraser" puck is available. This is acted by performing zigzag gestures on the element, as done with an actual eraser. Bridging the gap to the more natural approach of just grasping whatever you need from the bucket, to facilitate the process of adding blocks to the world, a tool is provided to clone blocks with a stamp as icon. By putting the cloner puck on a block, it will be able to replicate that block every time the puck is lifted up and put back down again.

Four additional tools are available. One is the modifier of friction. This allows the friction variation of a block with the surface in five discrete values ranging from 0 (no friction or highly polished material like glass) through infinite (rough and heavy material impossible to move). To change the friction value of a block, the puck has to be put on the block and be rotated until the value is selected (see Fig. 7). Once the selection is done the puck can be lifted up. As a common operation is to fix a block to the work surface, an alternative to the friction modifier tool is provided. This is the "locking/unlocking" tool. It allows specifying whether a block is static or not. The "plane/gravity" tool gives access to configure the expected gravity and the inclination of the plane in the simulation. Finally, the "play" tool controls the simulation of the world according to physics. The simulation is run by putting this puck on the surface. The simulation is finished, and the blocks are restored to the edition state, when the puck is lifted up.

\section{Method}

Test sessions were accommodated at the end of the course. Participants were assigned in sessions arranged on 2 days according to their availability limiting to 8 people per session. They were randomly grouped in pairs, but always taking into account age pairing. Each group got a colored card as identification.

Each group received an introductory talk about each experimentation platform. It was followed by a live demo of

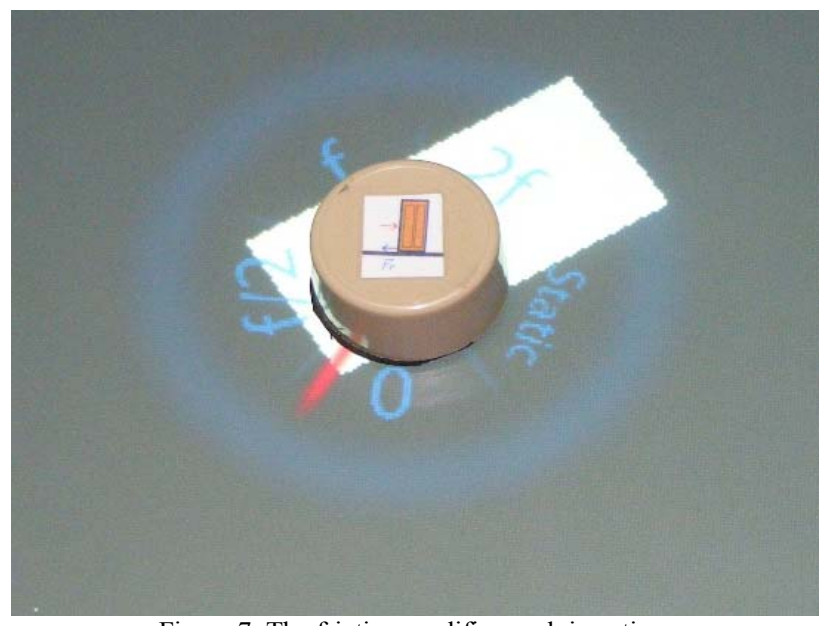

Figure 7. The friction modifier puck in action. 
how a given "hello world" problem could be solved using each platform, and finally they were required to give another solution to the demo problem. Their proposals were asked to be implemented in each platform by their own, providing a supervised interaction training to manage the platforms. All this took about 40 minutes.

After talks and training, they were introduced to the evaluation task (described below) and the activity began. The activity took place in two different places put in the loop: the thinking and the experimentation place. The places were separated by approximately two meters in order to avoid participants to watch what others were devising. First, group members produced solutions on paper so that they had a medium to support discussion to improve and generate new solutions collectively, and then they implemented those they believed to be more rewarding. The time for generating individual solutions was limited to a maximum of 10 minutes. The collective generation, and the implementation were also limited to 10 minutes. They were forced to pass to next stage when the time limit was reached.

Because of the time limitation available for experiments, the task being reported here could not last more than 60 minutes. Therefore each group only used an experimentation platform in the session. The designation of groups for one or another platform was done at random but always balancing their use.

Participants were encouraged to have good performance with two rewards for the best two groups. They were said to have good performance by producing a variety of solutions being as creative, original and elaborated as possible. Although they would not have enough time to implement and test their solutions in the experimentation platform they were reminded that it was important to give expression of as many solutions as they could on paper to promote divergent thinking and diversity of solutions.

All the interaction and manipulation on the experimentation platform was recorded on video for further analysis.

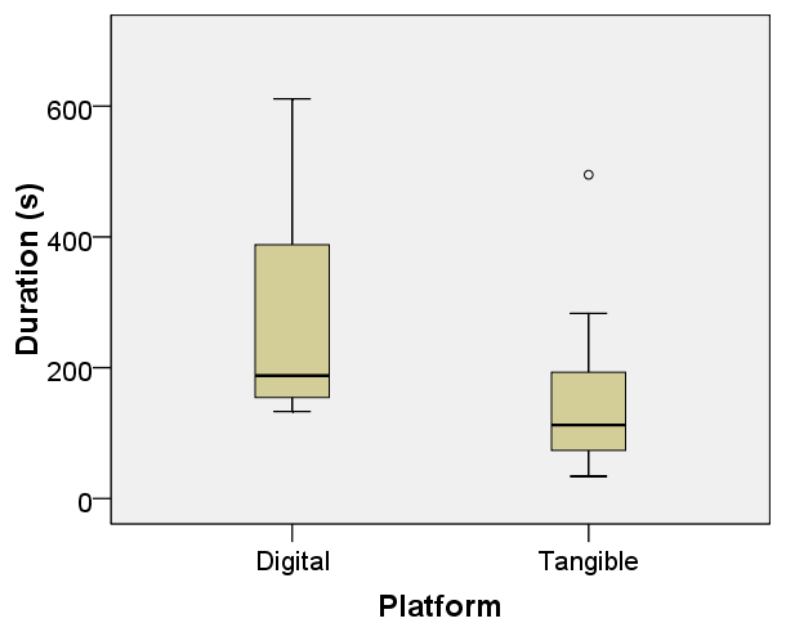

Figure 8. Box plot for time needed to test proposals.

\section{Task}

Participants were requested to produce as many solutions as possible to solve a proposed creative problem. In particular, the problem was to create entities with movable or articulated components. By "entity" it was meant anything, living entity or not, which could be represented with the material in the experimentation platforms. The creativity was not only expressed in the entities, but specially also in how they managed to make them movable by using joints.

When the working group was at the thinking place, they generated solutions. They always had to describe them by means of sketches using the solution forms (pencil and paper). Once each member had produced a bunch of solutions individually, they explained them to each other and discussed collectively about improvements and generation of new solutions. Then they decided what solutions to implement on the experimentation platform being assigned. Both activities, individual and collective generation of solutions, were limited to 10 minutes.

In the experimentation platform they had to implement their solutions. As they had discussed the ideas on paper, they already knew what parts of the entity needed to be constructed. Thus they could collaborate in order to finish faster. When the entity was built, they had to demonstrate to the experimenter that it worked as expected. Once they had tested all the solutions they had designed, or the 10 minutes limit was reached, they had to go back to the thinking place to start another cycle of reflection, discussion and action. The task finished when the time was up (60 minutes) regardless of which stage participants were in.

\section{E. Procedure}

Following the experiment design considerations, this task was only conducted once, and balanced designation of groups for using the experiment platform was carried out because of time limitations. Thus according to the designation, each group only interacted with a platform in the task being reported. The recordings were analyzed offline by the experimenter extracting information about performance on implementing solutions, their complexity,

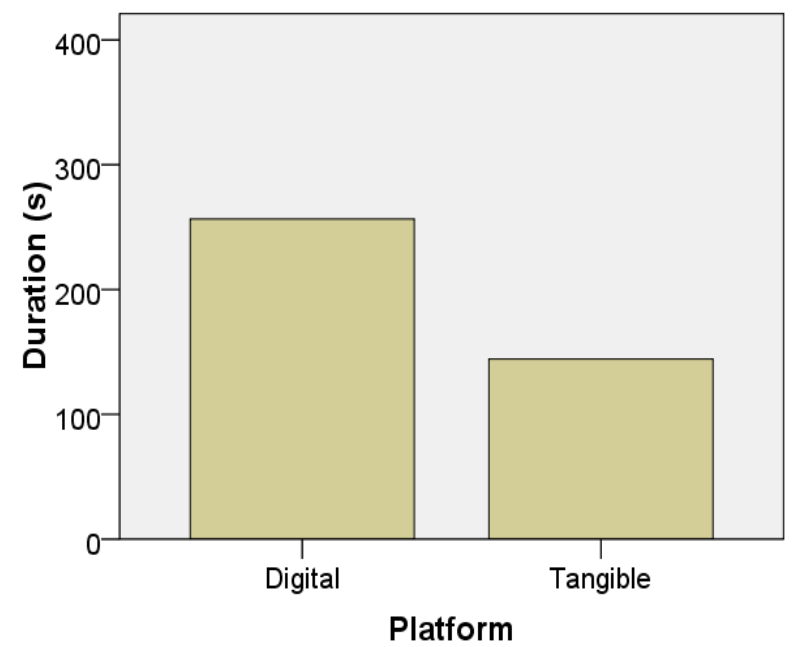

Figure 9. Mean plot for the time needed to test proposals. 


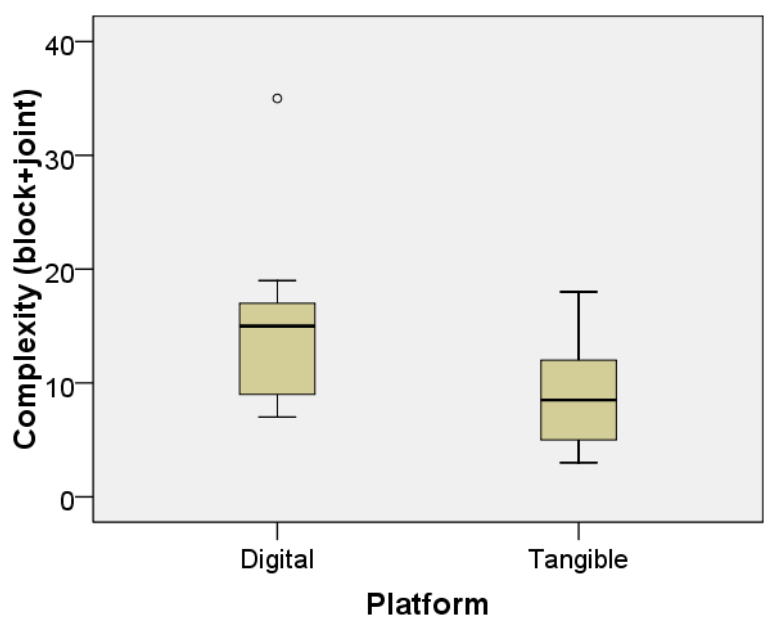

Figure 10. Box plot for the complexity of tested proposals. behavior patterns and collaboration degree.

\section{F. Results and Discussion}

During the task, the seven groups were able to design up to 98 proposals on paper, being only 44 finally tested in the assigned platform: the groups assigned to the digital platform tested 4 designs on average whereas the ones in the tangible platform tested 8 designs on average. This imbalance in the average number of proposals being tested per group depending on the platform is explained by the learning curve required to use the digital platform. This learning effect made the digital approach less productive in terms of the number of solutions obtained. To some extent this is expected because humans have been grasping and manipulating block-like elements since childhood, whereas new technologies always require learning, especially the ones with novel interaction techniques. Thus more training time should be accommodated to counteract this effect if a fair comparison wants to be carried out in terms of performance.

Fig. 8 shows the dispersion of the time needed to test a

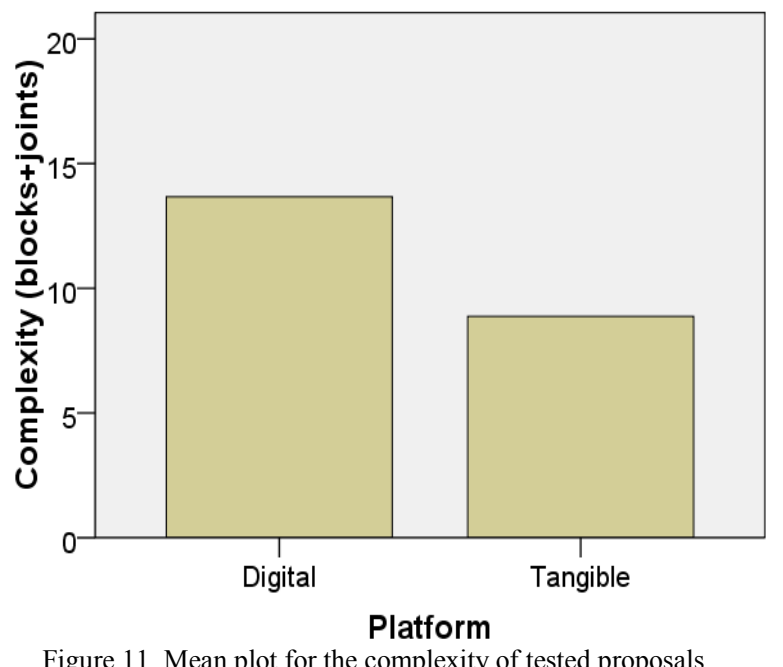

Figure 11. Mean plot for the complexity of tested proposals.

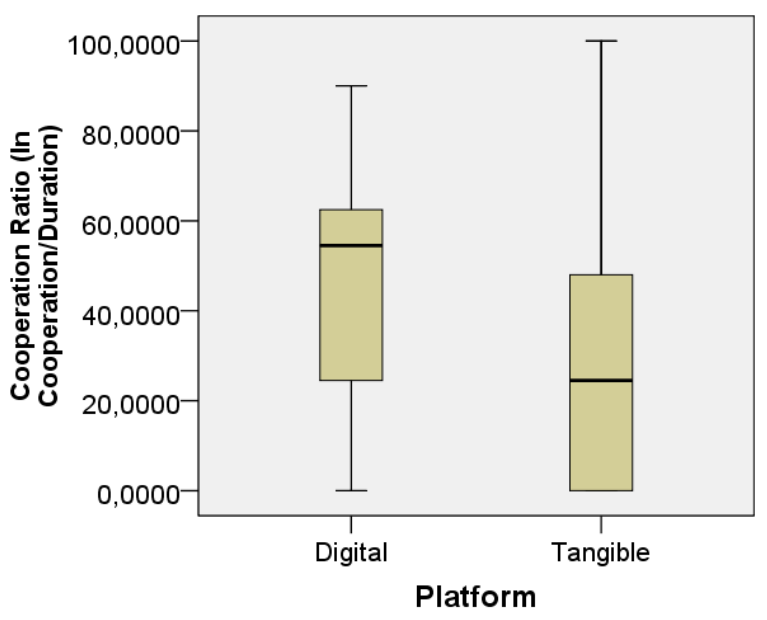

Figure 12. Box plot for the cooperation ratio

proposal by platform. On average, the testing of a proposal/solution in digital form took more time than in the tangible approach as shows Fig. 9. Specifically, the digital versions required 4 minutes and 16 seconds (i.e. 256.58s.) on average whereas the tangible ones required 2 minutes and 24 seconds (i.e. $144.25 \mathrm{~s}$.).

However, regarding the complexity of the solutions being proposed and finally tested, the study concludes that digital solutions are more complex on average (see Fig. 11). The digital solutions are more complex in both the number of blocks and number of joints being used. Fig. 10 shows the dispersion of complexity by platform. In terms of building blocks and joints involved in the tested proposals, the tangible solutions consist of nearly 9 elements $(\mathrm{M}=8.88$; $\mathrm{SD}=3.82)$ whereas the digital ones consist of $14(\mathrm{M}=13.67$; $\mathrm{SD}=8.41$ ).

This suggests that digital solutions require more time not only because of the learning-curve issue but also because the digital platform likely motivates users to consider more complex creations.

A priori both platforms are potentially good at sharing

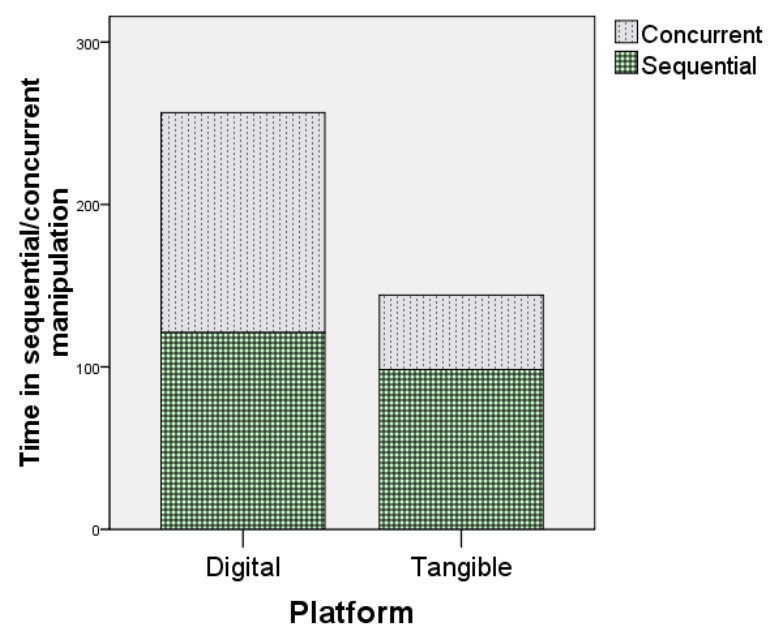

Figure 13. Proportions of concurrent and sequential interactions. 


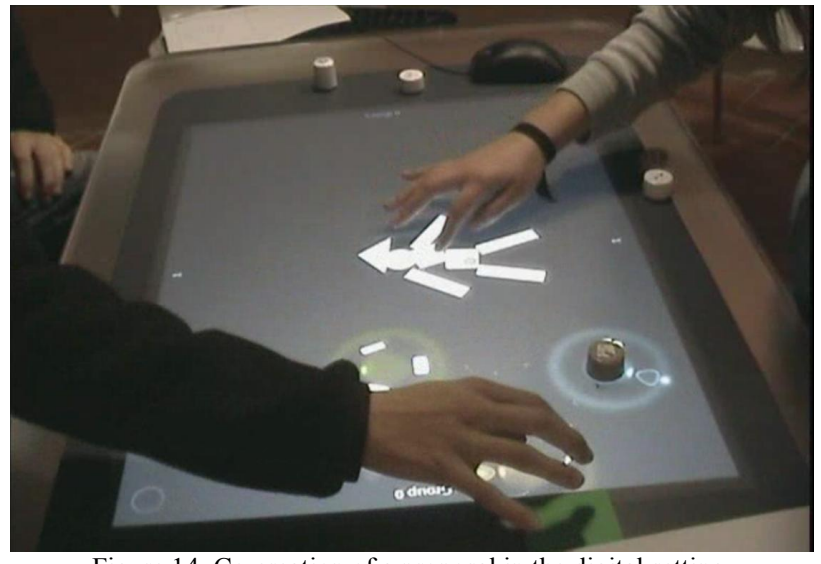

Figure 14. Co-creation of a proposal in the digital setting.

objects and supporting the collaborative task of creating entities with building blocks. This is especially important to know whether a platform is more suitable than the other to foster cooperative interaction and manipulation in co-located settings as the ones evaluated here. Taking this into consideration the recordings were analyzed in detail regarding the time in which members actually carried out cooperative work at the same time (i.e. concurrent manipulation of objects to create the entity). Fig. 12 shows the box plot for the cooperation ratio in the time needed to test proposals. On average, about $47 \%$ of the time was conducted in real cooperation when using the digital platform. The cooperation ratio dropped to $28 \%$ of the time in the tangible setting. This is consistent with the behavior of sequential or concurrent work observed in the recordings. Fig. 13 shows the average time consumed in sequential versus concurrent manipulation. There is a lot of sequential work in the tangible setting, typically with a member constructing the entity to some extent, then passing it to the other member to continue. Nevertheless an important amount of work is conducted in parallel in the digital platform. Fig. 14 shows the creation of a human-like entity in parallel in the digital setting.

This is an important result that raises the need to have a look into more cooperative behavior patterns to understand

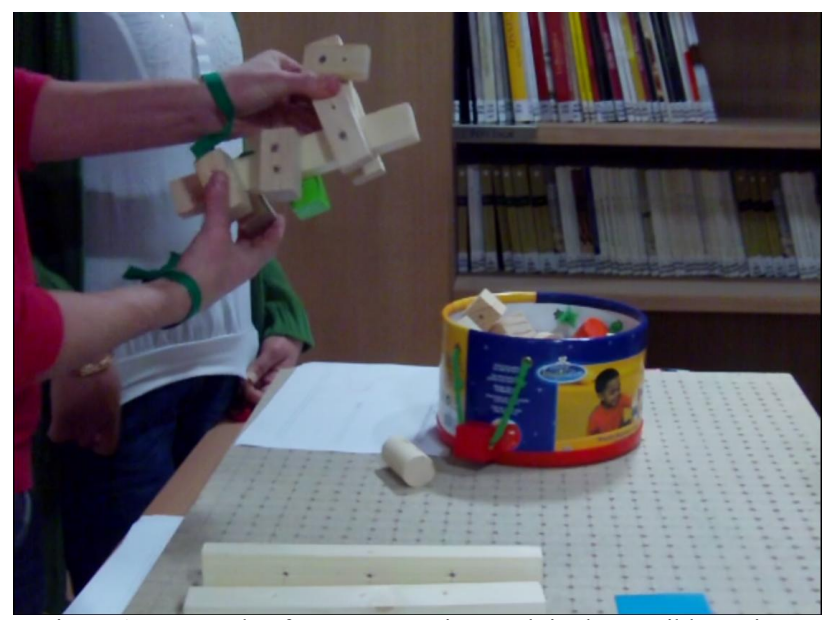

Figure 15. Example of non-cooperative work in the tangible setting. what actually is happening in each platform.

In both platforms the pattern provider-constructor was widely observed. From time to time one member acted as a provider of blocks and joints while the other focused on assembling them. In general, one member played the role of director/leader, and the other assumed a more passive role of follower. Normally the first role corresponded to the person that had designed the proposal at the thinking place. This is explained by the fact that they perceived the proposal as a property despite belonging to the group. However, in the digital platform the follower has a tendency towards being alert to get involved as soon as the other participant needs anything. Although this behavior is also present in the tangible approach, it is not as common, and participants just keep a block or joint at hand without any particular purpose. It rarely led to the participant testing blocks and joints to finally contribute to the creation.

In fact, it is remarkable that $37.5 \%$ of proposals tested in the tangible setup did not have any concurrent manipulation in cooperation (as in the situation illustrated in Fig. 15). This means that in 12 out 32 , a member created the entity without participation of his/her group mate, and normally without asking for help or even rejecting suggestions.

In the digital platform, this individualistic behavior only occurred once. This observation shows that humans have very present their notion of ownership when manipulating tangible elements. In this respect, in the tangible setting participants very often wanted to help by manipulating what the others were creating and the corresponding reactions were grasping the entity to their territory avoiding interruptions and cooperation. Related to this behavior, the analysis of the conversations revealed that if participants had very clear in mind how to implement the whole proposal, they preferred to work alone.

All these observations can be partially explained from the basic difference between both platforms. Both are tables, supporting co-operation, sharing objects and enabling faceto-face communication, but certainly the digital platform is the one that enforces keeping objects on the sharing space and allowing a more transparent management of territoriality and ownership. Although in the digital setting participants usually considered the nearest area of influence as their territory, common areas still remained available. However the tangible platform allowed the participants taking the entity under construction with them, to easily reflect human feelings related to ownership, individualism and noncooperative work.

Another interesting result is the rate of successful testing of previously designed solutions. While all the tested designs in the digital platform were successfully completed, it is remarkable that four entities were not completed in the tangible approach. From the recordings, the observation is that these unfinished creations were explained by either the complexity of the entity or the difficulty to join blocks as required. Participants tried to join pieces in many different ways, working sometimes sequentially but especially in parallel in two of these cases. In fact, the work in cooperation reached $95.5 \%$ and $57.2 \%$ respectively in these cases. Therefore, this is an important evidence of the 
outstanding increase of the concurrent cooperation when facing the implementation of complex or elaborated ideas. It is observed that the digital platform facilitates concurrent cooperation and composition of the different subparts of the entities by means of the flat sharing space. However, in the tangible approach cooperation patterns only arise when the complexity of the entity at hand is sufficiently high. Because participants usually take entities to their territory, the comanipulation becomes difficult and the entity has to be complex and large enough in order to enable each participant to create different parts collaboratively. Nevertheless, this way of cooperation is commonly and easily supported by the digital platform as the recordings have revealed.

\section{CONCLUSION AND FUTURE WORK}

This paper briefly presents the background and motivation for AGORAS, a new learning environment to support creative learning based on the creation of $2 \mathrm{D}$ ecosystems on interactive tabletops. A preliminary empirical study has been conducted to obtain initial insights about whether the grounding technology may become a promising tool in the field of creative learning and how results should impact on the ongoing development of the learning environment.

The study explores some aspects such as the productivity, complexity and concurrent co-manipulation of our interactive tabletop approach with respect to an equivalent traditional approach based on purely physical objects. These aspects are related to some important creativity attributes such as fluency or elaboration.

The results indicate that users are more productive in terms of the number of solutions obtained using a non computer-mediated approach for creating entities. However, using the digital tabletop approach users design, on average, more complex or elaborate solutions (in terms of the number of involved bodies and joints) and spend more time per entity in the testing/simulation space. The results also suggest that users in the digital approach have a higher success percentage, defined as the number of successfully completed, implemented and tested designs. This means the gap between conception on paper and creation in the simulation space is reduced when using a digital tabletop approach. Finally, the results show that teams establish more frequently cooperation schemes in the digital tabletop condition by sharing the simulation space. However in the pure tangible condition, ownership and self-manipulation interaction patterns arise and, as a result, sequential creative processes are observed.

These results reveal that tabletop based tools to support creativity or creative learning have a promising future and further creativity attributes and activities need to be explored. However the study has some limitations which we must be aware of. On the one hand, as a consequence of the limited time available to conduct the experiment within a course, the training was dramatically but necessarily shortened. On the other hand, this study must be considered exploratory, searching for initial insights because of the number of participants. Therefore, as findings seem promising enough despite these threats, the most immediate future work is to design and conduct an experiment with more participants and involving tasks of different nature. Probably, one similar to the presented here, which focuses on the creativity in designing and building entities, and another related to solving creative problems by designing RubeGoldberg machines.

By increasing the number of participants, and getting their school performance profiles beforehand, the empirical study will allow us to be more ambitious in scope. We will be able to group participants according to criteria from psychological and learning theories. In addition, more aspects related to creativity that have not still been studied here will be included, and conclusions will be drawn with a profounder data support, which will allow us to check if the preliminary outcomes obtained in this work are confirmed.

This experiment has served to proof a reduced entity editor prototype implemented for AGORAS and its grounding. In this sense, as the results are promising, we are already working on completing the prototype to be included in the full AGORAS learning environment aiming at running experiments with the fully functional version.

\section{ACKNOWLEDGMENT}

This work was funded by the Spanish Ministry of Education and Science and Innovation under the National Strategic Program of Scientific Research, Development and Technological Innovation $(\mathrm{I}+\mathrm{D}+\mathrm{i})$ and project TSI201020488. Our thanks to the Alaquas city council and the clubhouse's managers. Thanks also to the team Polimedia of the office "Área de Información y Comunicaciones" (ASIC) for the support in computer hardware.

A. Catalá is supported by a FPU fellowship from the Ministry of Education and Science of Spain with reference AP2006-00181.

\section{REFERENCES}

[1] Press release: "The education in Spain in numbers", in spanish: "Las cifras de la Educación en España. Estadísticas e indicadores. Edición 2008", Estadísticas de la Educación, Ministerio de Educación, Política Social y Deporte, 11 Octubre, 2007.

http://www.mepsyd.es/mecd/jsp/plantilla.jsp?id=3131\&area= estadisticas\&contenido=/estadisticas/educativas/cee/2007A/ce e-2007A.html

[2] E.A. Hanushek, "The Failure of Input-Based Schooling Policies," Economic Journal, Royal Economic Society, vol. 113(485), pp. F64-F98. 2003.

[3] A.J. Cropley, "Creativty in Education and Learning: A Guide for Teachers and Educators", Kogan Page, London, 2001.

[4] T.M. Amabile, "The social psychology of creativity". New York: Springer-Verlag, 1983.

[5] E. Hornecker, and J. Buur, "Getting a Grip on Tangible Interaction: A Framework on Physical Space and Social Interaction". Proceedings CHI 2006, pp.437-446, 2006.

[6] P. Marshall, Y. Rogers, and E. Hornecker, "Are Tangible Interfaces Really Any Better Than Other Kinds of Interfaces?", Workshop on Tangible User Interfaces in Context and Theory, ACM CHI 2007.

[7] J.M. Carroll, "Becoming social: Expanding scenario-based approaches in HCI". Behaviour and Information Technology, 15(4), 1996, 266-275. 
[8] D. J. Treffinger, G. C. Young, E. C. Selby, and C. Shepardson , "Assessing creativity: A guide for educators" (RM02170). Storrs, CT: The National Research Center on the Gifted and Talented, University of Connecticut, 2002.

[9] H. Gardner, "Creating minds". New York: Basic Books, 1993.

[10] M. Resnick, "Rethinking Learning in the Digital Age". In The Global Information Technology Report: Readiness for the Networked World, edited by G. Kirkman. Oxford University Press, 2002.

[11] G. Scott, L. Leritz, and M. Mumford, "The Effectiveness of Creativity Training: A Quantitative Review" in Creativity Research Journal; Lawrence Erlbaum Associates; 2004.

[12] M. Resnick, S. Ocko and S. Papert. "LEGO, Logo, and Design," Children's Environments Quarterly 5, no. 4, pp. 14$18,1988$.

[13] M. Resnick, F. Martin, S. Sargent and B. Silverman, "Programmable Bricks: Toys to Think With". IBM Systems Journal vol. 35, 1996, pp. 443-452.

[14] The PicoCricket website: http://www.picocricket.com/ Last visit on April 15, 2011.

[15] Lego MINDSTORMS NXT website: http://mindstorms.lego.com/ Last visit on April 15, 2011

[16] The RoboCup website, http://www.robocup.org/ Last visit on April 15, 2011.
[17] B. Ullmer and H. Ishii, "Emerging Frameworks for Tangible User Interfaces, In Human-Computer Interaction in the New Millenium", Ed. John M. Carroll, Addison-Wesley, pp.579$601,2001$.

[18] W. W. Gaver, J. Bowers, A. Boucher, H. Gellerson, S. Pennington, A. Schmidt, A. Steed, N. Villars and B. Walker, "The drift table: designing for ludic engagement", CHI '04 extended abstracts on Human factors in computing systems, ACM Press, pp.885-900, 2004.

[19] S. Jordà, M. Kaltenbrunner, G. Geiger and R. Bencina, "The reacTable*", Proceedings of the International Computer Music Conference (ICM05), 2005.

[20] S. Jordà, "On stage: the reactable and other musical tangibles go real", Int. J. Arts and Technology, Vol. 1, Nos. 3/4, 2008, pp.268-287.

[21] D. Gallardo, C. Fernàndez-Julià and S. Jordà, "TurTan: A Tangible programming language for creative exploration", Proceedings of the 2008 IEEE international workshop on horizontal interactive human-computer-systems, Amsterdam, The Netherlands, 2008.

[22] A. Catalá, and J. Jaen, "A semantic model for reactive entities to support collaborative game design". Proceedings of FuturePlay '08. ACM, pp. 216-219, 2008.

[23] The Farseer Physics Engine Website: http://farseerphysics.codeplex.com/ Last visit on April 15, 2011. 